\title{
Analysis and Management of Interference in 5G Reverse TDD HetNets
}

\author{
Luca Sanguinetti*†, Aris L. Moustakas $^{\dagger \ddagger}$ and Mérouane Debbah ${ }^{\dagger \S}$ \\ *Dipartimento di Ingegneria dell'Informazione, University of Pisa, Pisa, Italy \\ †Ecole supérieure d'électricité (Supélec), Gif-sur-Yvette, France

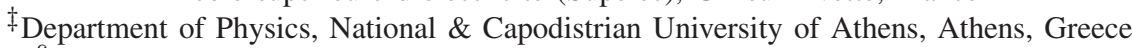 \\ $\S$ Mathematical and Algorithmic Sciences Lab, Huawei France R\&D, Paris, France
}

\begin{abstract}
This work analyzes a heterogeneous network (HetNet), which comprises a macro base station (BS) equipped with a large number of antennas and an overlaid dense tier of small cell access points (SCAs) using a wireless backhaul for data traffic. The static and low mobility user equipments (UEs) are associated with the SCAs while those with medium-to-high mobility are served by the macro BS. A reverse time division duplexing (TDD) protocol is used by the two tiers. We evaluate and characterize the performance of the system through the power consumption of uplink (UL) and downlink (DL) transmissions under the assumption that target rates must be satisfied and imperfect channel state information is available. The large system analysis is used to provide concise formulae for the asymptotic UL and DL transmit powers and precoding vectors under the above assumptions. Numerical results are used to validate the analysis in different settings and to make comparisons with alternative network architectures.
\end{abstract}

\section{INTRODUCTION}

In this work, we characterize and analyze the power consumption of an HetNet consisting of a massive MIMO macro tier overlaid with a second tier of small cell access points (SCAs) [1]-[3]. The user equipments (UEs) are endowed with a single antenna and have different speeds. Those that are static or have low mobility are associated with the SCAs while the medium-to-high mobility ones are served by the macro base station (BS). The excess antennas at the BS are used to serve the macro UEs (MUEs) and at the same time to play the role itself of wireless backhaul to the SCAs. The latter are divided in two groups such that the distance between SCAs belonging to the same group is maximized and the arising interference is mitigated. A similar division is performed on the MUEs on the basis of their proximity to the SCAs (see Fig. 1 of Section II). On the other hand, the interference between the macro and second tier (the so-called two-tier interference) is handled using a reverse time-division-duplexing (TDD) mode, i.e., the BS is in downlink (DL) mode when the SCAs operate in uplink (UL), and vice versa. A minimum-mean-square-error (MMSE) receiver is used in UL at the base station (BS) for interference mitigation while a concatenated linear precoding technique employing zero-forcing $(\mathrm{ZF})$ is used in DL to satisfy rate constraints and to null interference towards SCAs. The design and analysis of the network is performed under the

L. Sanguinetti is funded by the People Programme (Marie Curie Actions) FP7 PIEF-GA-2012-330731 Dense4Green. A. L. Moustakas is the holder of the DIGITEO "ASAPGONE" Chair. This research has also been supported by the FP7 NEWCOM\# (Grant no. 318306) and the ERC Starting MORE (Grant no. 305123). assumption of imperfect channel state information (CSI) for the MUEs (due to their mobility) and is conducted in the asymptotic regime where the number of BS antennas $N$ and the network size grow large with fixed ratio.

The main literature for the system under investigation and the proposed TDD protocol is represented by [4] wherein the authors propose a similar protocol to exploit the excess antennas at the BS for intra- and inter-tier interference reduction. In contrast to [4], a wireless backhaul is introduced here for the secondary tier and imperfect CSI is assumed for MUEs. The wireless backhaul forces us to modify the transmission protocol in [4] so as to account for reverse TDD not only between tiers but also between SCAs.

The wireless backhaul has also been recently considered in [3] and [5]. In [3], the authors focus on the scalability properties of a wireless backhaul network modelled as a random multi-antenna extended network. Geometric arguments are used to derive an information theoretic upper bound on the capacity of the network. In [5], a two-tier network is considered under the assumption that SCAs are full-duplex devices equipped with interference cancellation capabilities. A different line of research for wireless backhaul is in the context of mm-Wave communications [6].

The impact of imperfect CSI has been investigated in [7]. The analysis is conducted in the asymptotic regime and shows that the mobility of a UE has a detrimental effect on its own achievable rate, but has no direct impact on the other UEs. Instead, we consider a two-tier network and evaluate the impact of imperfect CSI on the power consumption in the UL and DL of each tier, while guaranteeing requested rates. Moreover, our analysis shows that orthogonal transmission resources should be allocated to highly mobile MUEs. A similar result has been pointed out in [8] and [9].

\section{SYSTEM MODEL}

We consider an HetNet $^{1}$ where a macro tier is augmented with a certain number of low range SCAs. Each SCA possesses a single antenna and devotes its available resources to its prescheduled small cell UE (SUE). The macro BS employs $N$

\footnotetext{
${ }^{1}$ The following notation is used throughout this work. Matrices and vectors are denoted by bold letters. The superscript ${ }^{\dagger}$ denotes hermitian operation and $|\mathcal{S}|$ is used to denote the cardinality of the enclosed set $\mathcal{S}$. We let $\mathbf{I}_{K}$ denote the $K \times K$ identity matrix, whereas $\mathbf{1}_{K}$ is the $K$-dimensional unit vector. We use $\mathcal{C N}(\cdot, \cdot)$ to denote a multi-variate circularly-symmetric complex Gaussian distribution whereas $\mathcal{N}(\cdot, \cdot)$ stands for a real one.
} 


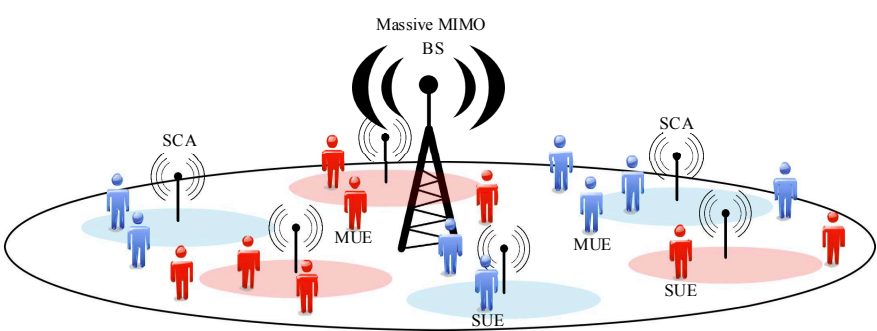

Fig. 1. Network architecture. The SCAs are divided in two different groups, namely, $\mathcal{S}_{\mathcal{B}}$ (blue colour) and $\mathcal{S}_{\mathcal{R}}$ (red colour). The same division is performed on the MUEs on the basis of their minimum distance from the SCAs.

transmit antennas to serve its associated single-antenna MUEs. As shown in Fig. 1, we assume that the SCAs are divided into two groups $\mathcal{S}_{\mathcal{R}}$ (red colour) and $\mathcal{S}_{\mathcal{B}}$ (blue colour) such that the distance between SCAs belonging to the same group is maximized. A similar division is performed on the MUEs on the basis of their proximity to the SCAs. We denote $\mathcal{M}_{\mathcal{R}}$ $\left(\mathcal{M}_{\mathcal{B}}\right)$ the set collecting MUEs that are closest to SCAs in $\mathcal{S}_{\mathcal{R}}\left(\mathcal{S}_{\mathcal{B}}\right)$. For notational convenience, we call $\mathcal{R}=\mathcal{M}_{\mathcal{R}} \cup \mathcal{S}_{\mathcal{R}}$ and $\mathcal{B}=\mathcal{M}_{\mathcal{B}} \cup \mathcal{S}_{\mathcal{B}}$.

The operating protocol is sketched in Fig. 2. In the frequency-time slot $\left(W_{1}, T_{1}\right)$, the MUEs and SCAs in $\mathcal{R}$ use the frequency band $W_{1}$ for UL transmissions (BS $\leftarrow$ MUE and $\mathrm{BS} \leftarrow \mathrm{SCA})$ for a time interval of length $T_{1}$ whereas the SCAs in $\mathcal{B}$ transmit to their associated SUEs in the DL (SCA $\rightarrow$ SUE). In $\left(W_{1}, T_{2}\right)$, the converse takes place. The frequency-time slots $\left(W_{2}, T_{1}\right)$ and $\left(W_{2}, T_{2}\right)$ are used in the dual way. As seen, the exchange of information within each tier takes place in a reverse order, i.e., the $\mathrm{BS}$ is in the DL mode (BS $\rightarrow$ MUE) when the SCAs operate in the UL (SCA $\leftarrow$ SUE), and vice versa. We also assume that $T_{1}+T_{2}$ is upper bounded by the coherence time of the channel. In these circumstances, UL and DL channels can be considered as reciprocal and the BS can make use of UL estimates for DL transmissions (more details on this will be given later on).

\section{A. Channel Model and Assumptions}

Let $\mathbf{h}_{i}^{\left(\mathcal{M}_{\mathcal{R}}\right)}=\left[h_{i}^{\left(\mathcal{M}_{\mathcal{R}}\right)}(1), h_{i}^{\left(\mathcal{M}_{\mathcal{R}}\right)}(2), \ldots, h_{i}^{\left(\mathcal{M}_{\mathcal{R}}\right)}(N)\right]^{T} \in$ $\mathbb{C}^{N \times 1}$ be the vector whose entry $h_{i}^{\left(\mathcal{M}_{\mathcal{R}}\right)}(n)$ accounts for the instantaneous propagation channel between the $i$ th MUE in $\mathcal{M}_{\mathcal{R}}$ and the $n$th antenna at the BS. Accordingly, we let $\mathbf{H}^{\left(\mathcal{M}_{\mathcal{R}}\right)}=\left[\mathbf{h}_{1}^{\left(\mathcal{M}_{\mathcal{R}}\right)} \mathbf{h}_{2}^{\left(\mathcal{M}_{\mathcal{R}}\right)} \cdots \mathbf{h}_{\left|\mathcal{M}_{\mathcal{R}}\right|}^{\left(\mathcal{M}_{\mathcal{R}}\right)}\right] \in \mathbb{C}^{N \times\left|\mathcal{M}_{\mathcal{R}}\right|}$ be the matrix collecting the channels of all MUEs in $\mathcal{M}_{\mathcal{R}}$. We assume that $\mathbf{h}_{i}^{\left(\mathcal{M}_{\mathcal{R}}\right)}=\sqrt{l\left(\mathbf{x}_{i}\right)} \mathbf{w}_{i}$ where $\mathbf{x}_{i}$ denotes the position of MUE $i$ in $\mathcal{M}_{\mathcal{R}}$ (computed with respect to the BS), $\mathbf{w}_{i} \sim \mathcal{C N}\left(0, \mathbf{I}_{N}\right)$ accounts for the small-scale fading channel and $l\left(\mathbf{x}_{i}\right): \mathbb{R}^{2} \rightarrow \mathbb{R}^{+}$is the average channel gain due to pathloss at distance $\left\|\mathbf{x}_{i}\right\|$. The same model is adopted for the channels of SCAs and SUEs in $\mathcal{S}_{\mathcal{R}}$ and $\mathcal{S}_{\mathcal{B}}$. In particular, we let $\mathbf{H}^{\left(\mathcal{S}_{\mathcal{R}}\right)} \in \mathbb{C}^{N \times\left|\mathcal{S}_{\mathcal{R}}\right|}$ and $\mathbf{H}^{\left(\mathcal{S}_{\mathcal{B}}\right)} \in \mathbb{C}^{N \times\left|\mathcal{S}_{\mathcal{B}}\right|}$ be the matrices collecting the channel gains from the BS antennas and the SCAs in $\mathcal{S}_{\mathcal{R}}$ and $\mathcal{S}_{\mathcal{B}}$, respectively.

In all subsequent discussions, we assume that imperfect CSI is available for MUEs. Observe that this naturally arises for MUEs as a consequence of mobility [7], [10]. In particular, we

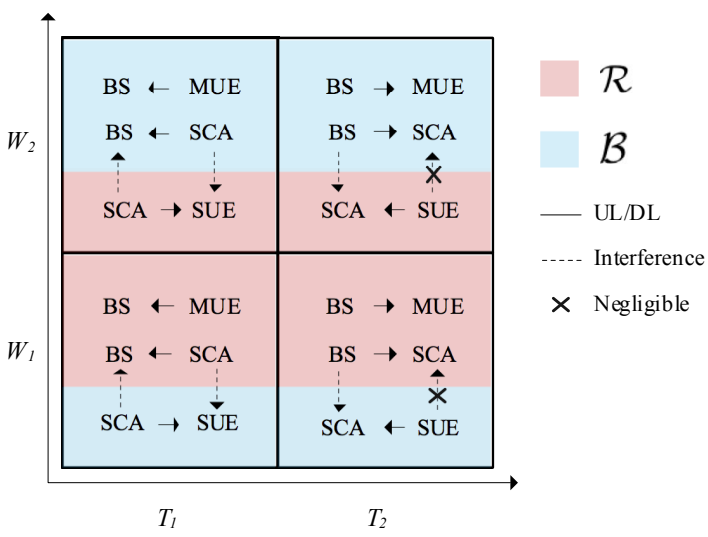

Fig. 2. Illustration of the transmission protocol. The exchange of information within each tier takes place in a reverse order, i.e., the BS is in the DL mode $(\mathrm{BS} \rightarrow \mathrm{MUE})$ when the SCAs operate in the $\mathrm{UL}(\mathrm{SCA} \leftarrow \mathrm{SUE})$, and vice versa.

let $\widehat{\mathbf{H}}^{\left(\mathcal{M}_{R}\right)}=\left[\widehat{\mathbf{h}}_{1}^{\left(\mathcal{M}_{R}\right)}, \widehat{\mathbf{h}}_{2}^{\left(\mathcal{M}_{R}\right)}, \ldots, \widehat{\mathbf{h}}_{\left|\mathcal{M}_{R}\right|}^{\left(\mathcal{M}_{R}\right)}\right]$ denote an estimate of $\mathbf{H}^{\left(\mathcal{M}_{\mathcal{R}}\right)}$ and assume that each vector $\widehat{\mathbf{h}}_{i}^{\left(\mathcal{M}_{\mathcal{R}}\right)}$ is modelled as $\widehat{\mathbf{h}}_{i}^{\left(\mathcal{M}_{\mathcal{R}}\right)}=\sqrt{l\left(\mathbf{x}_{i}\right)} \widehat{\mathbf{w}}_{i}$ with [10]

$$
\widehat{\mathbf{w}}_{i}=\sqrt{1-\tau_{i}^{2}} \mathbf{w}_{i}+\tau_{i} \mathbf{v}_{i}
$$

where $\mathbf{v}_{i} \sim \mathcal{C N}\left(0, \mathbf{I}_{N}\right)$ models the independent channel estimation errors. The parameter $\tau_{i} \in[0,1]$ reflects the accuracy or quality of the channel estimate $\widehat{\mathbf{h}}_{i}^{\left(\mathcal{M}_{R}\right)}$, i.e., $\tau_{i}=0$ corresponds to perfect CSI, whereas for $\tau_{i}=1$ the CSI is completely uncorrelated to the true channel.

Since SCAs occupy fixed positions in the network, in all subsequent discussions we assume that $\mathbf{H}^{\left(\mathcal{S}_{\mathcal{R}}\right)}$ and $\mathbf{H}^{\left(\mathcal{S}_{\mathcal{B}}\right)}$ are perfectly known at the BS. The same assumption is made for the SUE channels.

\section{LARGE SYSTEM ANALYSIS OF THE MACRO-TIER INTERFERENCE IN UL}

We start dealing with the case in which the BS is in UL mode. Without loss of generality, the frequency-time slot $\left(W_{1}, T_{1}\right)$ of Fig. 2 is considered. ${ }^{2}$ As seen, two instances of interference appear. One comes from UL signals of MUEs and SCAs in $\mathcal{R}$ and affects the receiving SUEs in $\mathcal{B}$ whereas the other accounts for the interference that the BS experiences from the DL mode of SCAs in $\mathcal{S}_{\mathcal{B}}$.

For notational convenience, we denote $K=|\mathcal{R}|$ the total number of transmitters (MUEs and SCAs) in $\mathcal{R}$ and call $S=$ $\left|\mathcal{S}_{\mathcal{B}}\right|$ the number of SCAs in $\mathcal{S}_{\mathcal{B}}$. We also let

$$
c=\frac{K}{N} \quad \text { and } \quad c_{S}=\frac{K+S}{N} .
$$

We let $\mathbf{H}=\left[\mathbf{h}_{1}, \mathbf{h}_{2}, \ldots, \mathbf{h}_{K}\right]=\left[\mathbf{H}^{\left(\mathcal{M}_{\mathcal{R}}\right)}, \mathbf{H}^{\left(\mathcal{S}_{\mathcal{R}}\right)}\right] \in \mathbb{C}^{N \times K}$ be the matrix collecting the instantaneous UL channels of MUEs and SCAs in $\mathcal{R}$ and denote $\left\{p_{k}^{(\mathcal{R}, \mathrm{ul})} \geq 0 ; k=\right.$ $1,2, \ldots, K\}$ the corresponding UL transmit powers. Letting $\mathbf{G}=\left[\mathbf{g}_{1}, \mathbf{g}_{2}, \ldots, \mathbf{g}_{K}\right] \in \mathbb{C}^{N \times K}$ be the MMSE matrix, the

\footnotetext{
${ }^{2}$ The same analysis can be performed for $\left(W_{2}, T_{1}\right)$.
} 


$$
\mathbf{G}=\widehat{\mathbf{H}}^{\dagger}\left(N \sigma^{2} \mathbf{I}_{N}+\sum_{i=1}^{K} p_{i}^{(\mathcal{R}, \mathrm{ul})} \widehat{\mathbf{h}}_{k} \widehat{\mathbf{h}}_{k}^{\dagger}+\sum_{s=1}^{S} p_{s}^{\left(\mathcal{S}_{\mathcal{B}}, \mathrm{dl}\right)} \mathbf{h}_{s}^{\left(\mathcal{S}_{\mathcal{B})}\right)} \mathbf{h}_{s}^{\left(\mathcal{S}_{\mathcal{B}}\right) \dagger}\right)^{-1}
$$

UL achievable rate for device $k$ in $\mathcal{R}$ is $R_{k}^{(\mathcal{R}, \mathrm{ul})}=\log _{2}(1+$ $\operatorname{SINR}_{k}^{(\mathcal{R}, \mathrm{ul})}$ ) with $\operatorname{SINR}_{k}^{(\mathcal{R}, \mathrm{ul})}$ given by

$$
\operatorname{SINR}_{k}^{(\mathcal{R}, \mathrm{ul})}=\frac{p_{k}^{(\mathcal{R}, \mathrm{ul})}\left|\mathbf{g}_{k}^{\dagger} \mathbf{h}_{k}\right|^{2}}{\mathcal{I}^{(\mathcal{R}, \mathrm{ul})}+\mathcal{I}^{\left(\mathcal{S}_{\mathcal{B}}, \mathrm{dl}\right)}+\sigma^{2}\left\|\mathbf{g}_{k}\right\|^{2}}
$$

where $\sigma^{2}$ accounts for thermal noise and

$$
\mathcal{I}^{(\mathcal{R}, \mathrm{ul})}=\sum_{i=1, i \neq k}^{K} p_{i}^{(\mathcal{R}, \mathrm{ul})}\left|\mathbf{g}_{k}^{\dagger} \mathbf{h}_{i}\right|^{2}
$$

and

$$
\mathcal{I}^{\left(\mathcal{S}_{\mathcal{B}}, \mathrm{dl}\right)}=\sum_{s=1}^{S} p_{s}^{\left(\mathcal{S}_{\mathcal{B}}, \mathrm{dl}\right)}\left|\mathbf{g}_{k}^{\dagger} \mathbf{h}_{s}^{\left(\mathcal{S}_{\mathcal{B}}\right)}\right|^{2}
$$

with $p_{s}^{\left(\mathcal{S}_{\mathcal{B}}, \mathrm{dl}\right)} \geq 0$ being the DL transmit power of SCA $s$ in $\mathcal{S}_{\mathcal{B}}$. As mentioned earlier, we assume that the MMSE receiver operates under the assumption of imperfect knowledge of $\mathbf{H}^{\left(\mathcal{M}_{\mathcal{R}}\right)}$. This amounts to setting $\mathbf{G}$ as shown in (2) where $\widehat{\mathbf{h}}_{k}$ is the $k$ th column of $\widehat{\mathbf{H}}$ given by $\widehat{\mathbf{H}}=\left[\widehat{\mathbf{H}}^{\left(\mathcal{M}_{\mathcal{R}}\right)} \mathbf{H}^{\left(\mathcal{S}_{\mathcal{R}}\right)}\right]$.

Remark 1. It is worth observing that in the frequencytime slot $\left(W_{1}, T_{1}\right)$ under consideration $\mathbf{H}^{\left(\mathcal{S}_{\mathcal{R}}\right)}$ can be easily acquired at the BS using UL pilots from SCAs in $\mathcal{S}_{\mathcal{R}}$. On the other hand, the estimation of $\mathbf{H}^{\left(\mathcal{S}_{\mathcal{B}}\right)}$ must be performed in a different way since the UL mode $(B S \leftarrow S C A)$ for $\mathcal{S}_{\mathcal{B}}$ takes place over the frequency band $W_{2}$. A possible solution might consist in using pilots that the SCAs in $\mathcal{S}_{\mathcal{B}}$ send in $D L(S C A \rightarrow S U E)$ to their associated SUEs. An alternative approach might be to periodically switch the operations of frequency bands $W_{1}$ and $W_{2}$ [11].

The aim of this section is to compute the UL and DL transmit powers $\left\{p_{k}^{(\mathcal{R}, \mathrm{ul})} \geq 0\right\}$ and $\left\{p_{s}^{\left(\mathcal{S}_{\mathcal{B}}, \mathrm{dl}\right)} \geq 0\right\}$ required to meet the corresponding rate constraints $\left\{r_{k}^{(\mathcal{R}, \text { ul })} ; k=1,2, \ldots, K\right\}$ and $\left\{r_{s}^{\left(\mathcal{S}_{\mathcal{B}}, \mathrm{dl}\right)} ; s=1,2, \ldots, S\right\}$ under imperfect CSI of MUEs. For notational convenience, the superscripts ${ }^{(\mathrm{ul})}$ and ${ }^{(\mathrm{dl})}$ are dropped in the sequel. The following lemma can be proved.

Lemma 1 ([12]). If the MMSE receiver is employed at the $B S$, then in the limit $N, K, S \rightarrow \infty$ with fixed $c \in(0,1]$ and $c_{S} \in(0,1]$ we have $p_{k}^{(\mathcal{R})}-\bar{p}_{k}^{(\mathcal{R})} \stackrel{a . s}{\longrightarrow} 0$ and $p_{s}^{\left(\mathcal{S}_{\mathcal{B}}\right)}-\bar{p}_{s}^{\left(\mathcal{S}_{\mathcal{B}}\right)} \stackrel{a . s_{.}}{\longrightarrow} 0$ where

$$
\bar{p}_{k}^{(\mathcal{R})}=\frac{1}{\mu \delta} \frac{\gamma_{k}}{l\left(\mathbf{x}_{k}\right)\left(1-\tau_{k}^{2}\right)}
$$

and

$$
\bar{p}_{s}^{\left(\mathcal{S}_{\mathcal{B}}\right)}=\frac{\gamma_{s}}{l\left(\mathbf{x}_{s}\right)}\left(\sigma^{2}+\frac{1}{\mu \delta} \sum_{k=1}^{K} \frac{\gamma_{k}}{1-\tau_{k}^{2}} \frac{l\left(\mathbf{x}_{k, s}\right)}{l\left(\mathbf{x}_{k}\right)}\right)
$$

with $\tau_{k}=0$ if $k \in \mathcal{S}_{\mathcal{R}}$ and $\gamma_{k}=2^{r_{k}^{(\mathcal{R})}}-1$. The quantities $\mu$ and $\delta$ are computed as the unique solutions to the two equations given by

$$
\mu \sigma^{2}+\frac{1}{N} \sum_{k=1}^{K} \frac{\gamma_{k}}{\delta\left(1-\tau_{k}^{2}\right)+\gamma_{k}}+\frac{1}{N} \sum_{s=1}^{S} \frac{p_{s}^{\left(\mathcal{S}_{\mathcal{B}}\right)} l\left(\mathbf{x}_{s}\right) \mu}{1+p_{s}^{\left(\mathcal{S}_{\mathcal{B}}\right)} l\left(\mathbf{x}_{s}\right) \mu}=1
$$

and (10).

As seen, the computation of $\left\{p_{k}^{(\mathcal{R})}\right\}$ and $\left\{p_{s}^{\left(\mathcal{S}_{\mathcal{B}}\right)}\right\}$ reduces to the easy task of finding $\mu$ and $\delta$ as the unique solutions of a set of equations that depend only on system parameters.

From the above results, it follows that the imperfect CSI coefficients $\left\{\tau_{k}\right\}$ impact on both $\mu$ and $\delta$ in (9) and (10). In particular, from (7) it follows that $1 / \delta$ can be thought of as the fractional UL power increase of all transmitters (MUEs and $\mathrm{SCAs}$ ) in $\mathcal{R}$. Interestingly, this happens even though only the MUE channels are estimated erroneously while perfect CSI is assumed for SCAs. More precisely, it turns out that $\delta \rightarrow 0$ and thus $p_{k}^{(\mathcal{R})} \rightarrow \infty \forall k$ when [12]

$$
\frac{1}{N} \sum_{k \in \mathcal{M}_{\mathcal{R}}} \gamma_{k} \frac{\tau_{k}^{2}}{1-\tau_{k}^{2}} \geq 1-c_{S}
$$

meaning that there exist critical values for $\left\{\tau_{k}\right\}$ and $\left\{\gamma_{k}\right\}$ beyond which all powers diverge. If $\tau_{k}=\tau$ for any $k \in \mathcal{M}_{\mathcal{R}}$ one gets that $\tau$ has to be smaller than $\tau_{\text {MMSE }}^{\text {(max) }}$ given by

$$
\tau_{\mathrm{MMSE}}^{(\max )}=\left(1+\gamma^{\left(\mathcal{M}_{\mathcal{R}}\right)} \frac{c}{1-c_{S}}\right)^{-1 / 2}
$$

where $\gamma^{\left(\mathcal{M}_{\mathcal{R}}\right)}=\frac{1}{K} \sum_{k \in \mathcal{M}_{\mathcal{R}}} \gamma_{k}$.

\section{LARge SySTEM ANALYSIS OF THE MACRO-TIER INTERFERENCE IN DL}

We now consider the case in which the BS is in DL mode. Without loss of generality, the frequency-time slot $\left(W_{1}, T_{2}\right)$ of Fig. 2 is considered. As for the UL, two instances of interference arise. The interference experienced by MUEs and SCAs from UL transmissions in $\mathcal{S}_{\mathcal{B}}$ can be reasonably neglected since the number of transmitting SUEs is relatively small (one per SCA) and geographically far away from the MUEs and SCAs in $\mathcal{R}$. On the other hand, the interference from BS to the SCAs in UL must be properly mitigated to avoid a severe degradation of the network performance. For this purpose, we assume that the BS makes use of linear precoding and sacrifices some of its degrees of freedom (or excess antennas) to simultaneously serve all receivers in $\mathcal{R}$ and at the same time to null the interference towards $\mathcal{S}_{\mathcal{B}}$. We let $\mathbf{V}=\left[\mathbf{v}_{1}, \mathbf{v}_{2}, \ldots, \mathbf{v}_{K}\right] \in \mathbb{C}^{N \times K}$ be the precoding matrix and denote $p_{k}^{(\mathcal{R}, \mathrm{dl})}$ the DL transmit power assigned to the $k$ th device in $\mathcal{R}$. The total DL transmit power at the BS is thus 


$$
\delta=\left(1+\frac{\frac{1}{N} \sum_{k=1}^{K} \frac{\gamma_{k}}{\delta} \frac{\tau_{k}^{2}}{1-\tau_{k}^{2}}}{\mu \sigma^{2}+\frac{1}{N} \sum_{k=1}^{K} \frac{\gamma_{k} \delta\left(1-\tau_{k}^{2}\right)^{2}}{\left(\delta\left(1-\tau_{k}^{2}\right)+\gamma_{k}\right)^{2}}+\frac{1}{N} \sum_{s=1}^{S} \frac{p_{s}^{\left(\mathcal{S}_{\mathcal{B}}\right)} l\left(\mathbf{x}_{s}\right) \mu}{\left(1+p_{s}^{\left(\mathcal{S}_{\mathcal{B}}\right)} l\left(\mathbf{x}_{s}\right) \mu\right)^{2}}}\right)^{-1} .
$$

given by

$$
P^{(\mathcal{R}, \mathrm{dl})}=\sum_{k=1}^{K} p_{k}^{(\mathcal{R}, \mathrm{dl})}\left\|\mathbf{v}_{k}\right\|^{2} .
$$

The achievable DL rate for a generic receiver $k$ in $\mathcal{R}$ takes the form $R_{k}^{(\mathcal{R}, \mathrm{dl})}=\log _{2}\left(1+\operatorname{SINR}_{k}^{(\mathcal{R}, \mathrm{dl})}\right)$ with

$$
\operatorname{SINR}_{k}^{(\mathcal{R}, \mathrm{dl})}=\frac{p_{k}^{(\mathcal{R}, \mathrm{dl})}\left|\mathbf{h}_{k}^{\dagger} \mathbf{v}_{k}\right|^{2}}{\sum_{i=1, i \neq k}^{K} p_{i}^{(\mathcal{R}, \mathrm{dl})}\left|\mathbf{h}_{k}^{\dagger} \mathbf{v}_{i}\right|^{2}+\sigma^{2}} .
$$

We denote $r_{k}^{(\mathcal{R}, \mathrm{dl})}$ the DL target rate of the $k$ th receiver in $\mathcal{R}$. Imposing $R_{k}^{(\mathcal{R}, \mathrm{dl})}=r_{k}^{(\mathcal{R}, \mathrm{dl})}$ amounts to setting $\operatorname{SINR}_{k}^{(\mathcal{R}, \mathrm{dl})}=$ $\gamma_{k}$ with $\gamma_{k}$ given by $\gamma_{k}=2^{r_{k}^{(\mathcal{R}, \mathrm{dl})}}-1$. Thanks to the reciprocity of UL and DL channels, the BS can exploit UL estimates for DL transmissions. As for the UL, we assume that perfect knowledge of $\mathbf{H}^{\left(\mathcal{S}_{\mathcal{B}}\right)}$ and $\mathbf{H}^{\left(\mathcal{S}_{\mathcal{R}}\right)}$ is available while imperfect CSI is assumed for $\mathbf{H}^{\left(\mathcal{M}_{\mathcal{R}}\right)}$. For notational convenience, the superscript ${ }^{(\mathrm{dl})}$ is dropped in the sequel.

The complete elimination of the macro-tier interference at SCAs in $\mathcal{S}_{\mathcal{B}}$ can be achieved by constraining the precoding matrix $\mathbf{V}$ to lie in the null space of $\mathbf{H}^{\left(\mathcal{S}_{\mathcal{B}}\right)}$. Under the assumption of perfect knowledge of $\mathbf{H}^{\left(\mathcal{S}_{\mathcal{B}}\right)}$, this is achieved setting $\mathbf{V}=\mathbf{T}^{\left(\mathcal{S}_{\mathcal{B})}\right)} \mathbf{F}$ where $\mathbf{F}=\left[\mathbf{f}_{1}, \mathbf{f}_{2}, \ldots, \mathbf{f}_{K}\right] \in \mathbb{C}^{N \times K}$ is a design matrix and $\mathbf{T}^{\left(\mathcal{S}_{\mathcal{B}}\right)}=\mathbf{I}_{N}-\mathbf{H}^{\left(\mathcal{S}_{\mathcal{B}}\right)}\left(\mathbf{H}^{\left(\mathcal{S}_{\mathcal{B}}\right)^{\dagger}} \mathbf{H}^{\left(\mathcal{S}_{\mathcal{B}}\right)}\right)^{-1} \mathbf{H}^{\left(\mathcal{S}_{\mathcal{B}}\right)^{\dagger}} \in$ $\mathbb{C}^{N \times N}$. Let $\mathbf{U}=\mathbf{T}^{\left(\mathcal{S}_{\mathcal{B})}\right)} \mathbf{H} \in \mathbb{C}^{N \times K}$ be the composite channel and denote $\widehat{\mathbf{U}}$ its corresponding estimate defined as $\widehat{\mathbf{U}}=\mathbf{T}^{\left(\mathcal{S}_{\mathcal{B}}\right)} \widehat{\mathbf{H}}$ where $\widehat{\mathbf{H}}$ is given by $\widehat{\mathbf{H}}=\left[\widehat{\mathbf{H}}^{\left(\mathcal{M}_{R}\right)} \mathbf{H}^{\left(\mathcal{S}_{R}\right)}\right]$. The matrix $\widehat{\mathbf{U}}$ is used in the sequel to design $\mathbf{F}$ according to the $\mathrm{ZF}$ criterion. This yields

$$
\mathbf{V}_{\mathrm{ZF}}=\mathbf{T}^{\left(\mathcal{S}_{\mathcal{B}}\right)} \widehat{\mathbf{H}}\left(\widehat{\mathbf{H}}^{\dagger} \mathbf{T}^{\left(\mathcal{S}_{\mathcal{B}}\right)} \widehat{\mathbf{H}}\right)^{-1} .
$$

Lemma 2 ([12]). If $Z F$ is used and $K, N, S \rightarrow \infty$ with $c_{\mathcal{S}} \in$ $(0,1]$, then $P_{Z F}^{(\mathcal{R})}-\bar{P}_{Z F}^{(\mathcal{R})} \stackrel{\text { a.s }}{\longrightarrow} 0$ where

$$
\bar{P}_{Z F}^{(\mathcal{R})}=c \sigma^{2} \frac{A}{1-c_{\mathcal{S}}-c B} .
$$

Also, $p_{k}^{(\mathcal{R})}-\bar{p}_{k}^{(\mathcal{R})} \stackrel{\text { a.s. }}{\longrightarrow} 0$ where

$$
\bar{p}_{k}^{(\mathcal{R})}=\frac{\gamma_{k}}{1-\tau_{k}^{2}}\left(\sigma^{2}+\tau_{k}^{2} l\left(\mathbf{x}_{k}\right) \bar{P}_{Z F}^{(\mathcal{R})}\right)
$$

with $\tau_{k}=0$ if $k \in \mathcal{S}_{\mathcal{R}}$.

Since $\bar{P}_{\mathrm{ZF}}$ must be positive and finite, it follows that the asymptotic analysis can be applied to ZF only when the following condition is satisfied: $1-c_{S}-c B>0$ or, equivalently,

$$
\frac{1}{K} \sum_{k \in \mathcal{M}_{\mathcal{R}}}^{K} \gamma_{k} \frac{\tau_{k}^{2}}{1-\tau_{k}^{2}}<\frac{1-c_{\mathcal{S}}}{c} .
$$

If $\tau_{k}=\tau$ for any $k$, then we have that

$$
\gamma^{\left(\mathcal{M}_{\mathcal{R}}\right)}<\frac{1-c_{\mathcal{S}}}{c} \frac{1-\tau^{2}}{\tau^{2}}
$$

from which it follows $\tau<\tau_{\mathrm{ZF}}^{(\max )}$ with

$$
\tau_{\mathrm{ZF}}^{(\max )}=\left(1+\gamma^{\left(\mathcal{M}_{\mathcal{R}}\right)} \frac{c}{1-c_{S}}\right)^{-1 / 2} .
$$

Observe that the same condition must be fulfilled in the UL (see (12) in Section III).

\section{Numerical Results}

The accuracy of the above asymptotic characterization is now validated numerically by Monte-Carlo simulations. The results are obtained for 1000 different channel realizations and UE distributions. We assume that the BS is equipped with $N=128$ antennas and covers a square area centered at the BS with side length $500 \mathrm{~m}$ over which 16 SCAs are distributed on a regular grid with an inter-site distance of $125 \mathrm{~m}$. We assume that 128 MUEs are active in the cell and that a single SUE is uniformly distributed within a disc of radius $35 \mathrm{~m}$ around each SCA. The SUEs are associated with the closest SCA while the MUEs are associated with the BS. Accordingly, the two sets $\mathcal{R}$ and $\mathcal{B}$ count 64 MUEs and 8 SCAs with 8 MUEs in the proximity of each SCA. We assume that the UL and DL wireless backhaul rates of SCAs $r_{s}^{\left(\mathcal{S}_{\mathcal{B}}, \mathrm{dl}\right)}$ and $r_{s}^{\left(\mathcal{S}_{\mathcal{B}}, \mathrm{ul}\right)}$ are equal and fixed to $3 \mathrm{bit} / \mathrm{s} / \mathrm{Hz}$. The pathloss function $l(\mathbf{x})$ is modelled as $l(\mathbf{x})=2 L_{\bar{x}}\left(1+\|\mathbf{x}\|^{\beta} / \bar{x}^{\beta}\right)^{-1}$ where $\beta \geq 2$ is the pathloss exponent, $\bar{x}>0$ is some cut-off parameter and $L_{\bar{x}}$ is a constant that regulates the attenuation at distance $\bar{x}$. We assume that $\beta=3.5$ and $L_{\bar{x}}=-86.5 \mathrm{~dB}$ [13], [14]. Although in TDD systems the effective values of $\left\{\tau_{k}\right\}$ are expected to be different between UL and DL (since the channels are estimated in the UL and then used in DL), the same values of $\left\{\tau_{k}\right\}$ are used for both links in all subsequent simulations. The parameter setting is summarized in Table I for simplicity. Comparisons are made with two alternative networks [12]. In particular, we consider a HetNet in which the SCAs use a wired backhaul infrastructure for data traffic and a massive MIMO system in which all UEs (MUEs and SUEs) are served by the macro BS.

Fig. 3 depicts the average transmit power for MUEs and SCAs over the cell as a function of the requested rate of MUEs 
TABLE I

\begin{tabular}{|c|c|c|c|}
\hline Parameter & Value & Parameter & Value \\
\hline Bandwidth & $W=10 \mathrm{MHz}$ & Total number of SCAs & 16 \\
\hline Noise power & $W \sigma^{2}=-104 \mathrm{dBm}$ & Small-cell radius & $R=35 \mathrm{~m}$ \\
\hline Macro-cell side length & $500 \mathrm{~m}$ & Inter-side distance of SCAs & $\Delta=125 \mathrm{~m}$ \\
\hline Cut-off parameter & $\bar{x}=25 \mathrm{~m}$ & Pathloss coefficient & $\beta=3.5$ \\
\hline Carrier frequency & $f_{c}=2.4 \mathrm{GHz}$ & Average pathloss attenuation at $\bar{x}$ & $L_{\bar{x}}=-86.5 \mathrm{~dB}$ \\
\hline Number of BS antennas & $N=128$ & Imperfect CSI for MUEs & $\tau^{2}=0,0.1$ and 0.3 \\
\hline Total number of MUEs & 128 & Wireless Backhaul Requirements for SCAs & $r_{s}=3 \mathrm{bit} / \mathrm{s} / \mathrm{Hz}$ \\
\hline
\end{tabular}

UL power for MUEs and SCAs for different network architectures

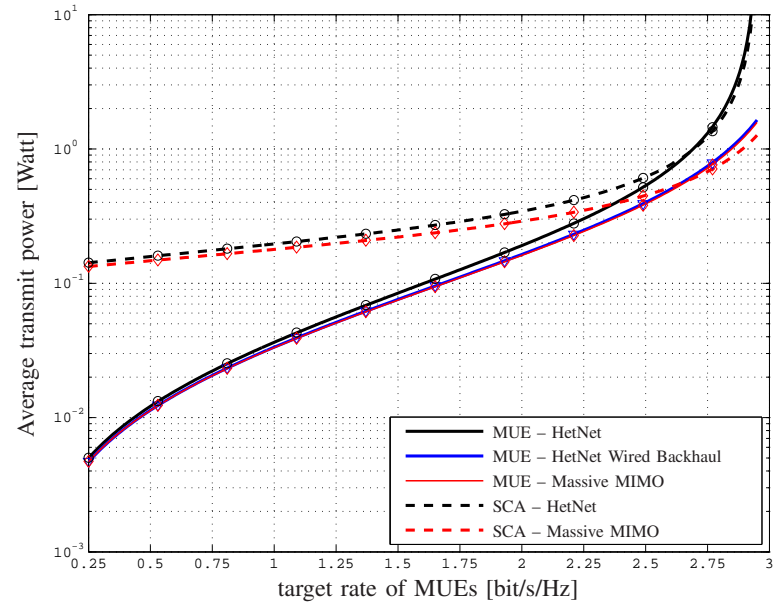

Fig. 3. Average UL transmit power for MUEs and SCAs as a function of MUE rates for different network architectures when $\tau^{2}=0.1$ and the wireless backhaul traffic is fixed to $3 \mathrm{bit} / \mathrm{s} / \mathrm{Hz}$.

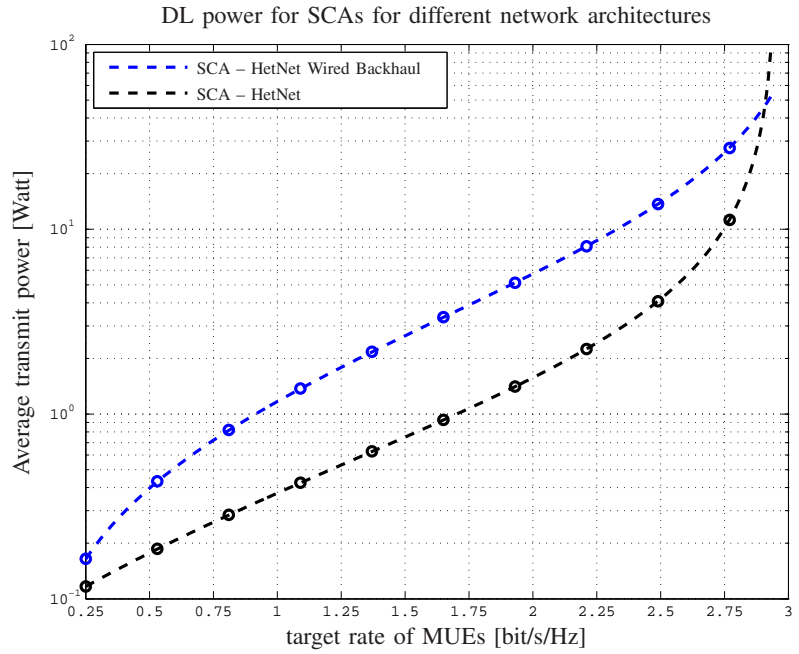

Fig. 4. Average DL transmit power for SCAs towards their respective SUEs as a function of MUE rates for the HetNet and HetNet with wired Backhaul architectures when $\tau^{2}=0.1$ and the wireless backhaul traffic is fixed to 3 $\mathrm{bit} / \mathrm{s} / \mathrm{Hz}$.

when $\tau^{2}=0.1$. Despite the fact that the power of SCAs in (7) does not depend explicitly on the MUE rates $r_{k}^{(\mathcal{R}, \mathrm{ul})}$ for $k \in \mathcal{M}_{\mathcal{R}}$, a mild dependence on MUE requirements is shown in the results of Fig. 3. This is due to the fact that $\mu$ and $\delta$ in (9) and (10) depend on $\left\{r_{k}^{(\mathcal{R}, \mathrm{ul})}\right\}$ through $\left\{\gamma_{k}\right\}$. A similar behaviour is observed in Fig. 4 for the DL power of SCAs. From the results of Fig. 3, it also follows that the average UL power of MUEs in the proposed HetNet is substantially the

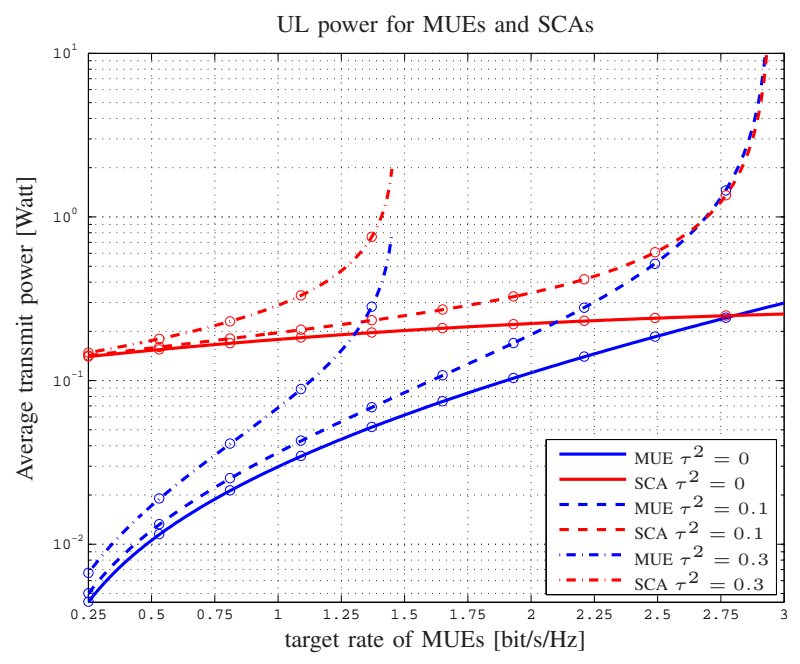

Fig. 5. Average UL transmit power for MUEs and SCAs for the proposed HetNet architecture as a function of MUE rates for different values of $\tau^{2}$. The wireless backhaul traffic is set to $3 \mathrm{bit} / \mathrm{s} / \mathrm{Hz}$.

same of a HetNet with wired backhaul even though a wireless backhaul traffic of $3 \mathrm{bit} / \mathrm{s} / \mathrm{Hz}$ is provided. On the contrary, Fig. 4 shows a significant power reduction for SCAs in DL mode.

Figs. 5 provides insights on the effect of channel uncertainty on power consumption. Clearly, $\tau^{2}=0$ corresponds to the perfect CSI case. It can be seen that for $\tau^{2}=0.3$ (corresponding to a velocity of $50 \mathrm{~km} / \mathrm{h}$ ) the system becomes infeasible if the target rates go beyond a certain level given approximately by $1.5 \mathrm{bit} / \mathrm{s} / \mathrm{Hz}$ (as obtained through (12)). Indeed, the power of every transmitter becomes infinite. However, this happens for a relatively narrow window of rate values, thereby allowing the system to operate at relatively low powers up to the critical values. In particular, we observe that for $\tau^{2}=0.1$ each MUE requires $80 \mathrm{~mW}$ to achieve a target rate of $1.5 \mathrm{bit} / \mathrm{s} / \mathrm{Hz}$. Taking into account that the bandwidth is $W=10 \mathrm{MHz}$ and the number of MUEs for frequency-time slot is 64 MUEs, this corresponds to an aggregate area throughput of $3.94 \mathrm{~Gb} / \mathrm{s} / \mathrm{km}^{2}$.

Fig. 6 illustrates the average DL transmit power of the BS when $\mathrm{ZF}$ is employed with $\tau^{2}=0.1$. In particular, we observe that for a target rate of $2 \mathrm{bit} / \mathrm{s} / \mathrm{Hz}$ only $0.1 \mathrm{~W}$ are required at the BS to serve (in DL) all MUEs and SCAs. This corresponds to an area throughput of $5.12 \mathrm{Gbit} / \mathrm{s} / \mathrm{km}^{2}$. Compared to the two alternative network configurations, a marginal increase of power is required by the proposed HetNet with both precoding techniques. However, this is achieved at the price of a substantial power saving at the SUEs. Indeed, numerical results reveal that for a target rate of $3 \mathrm{bit} / \mathrm{s} / \mathrm{Hz}$, the required power for a SUE is $0.85 \mathrm{~mW}$ in the HetNet case 


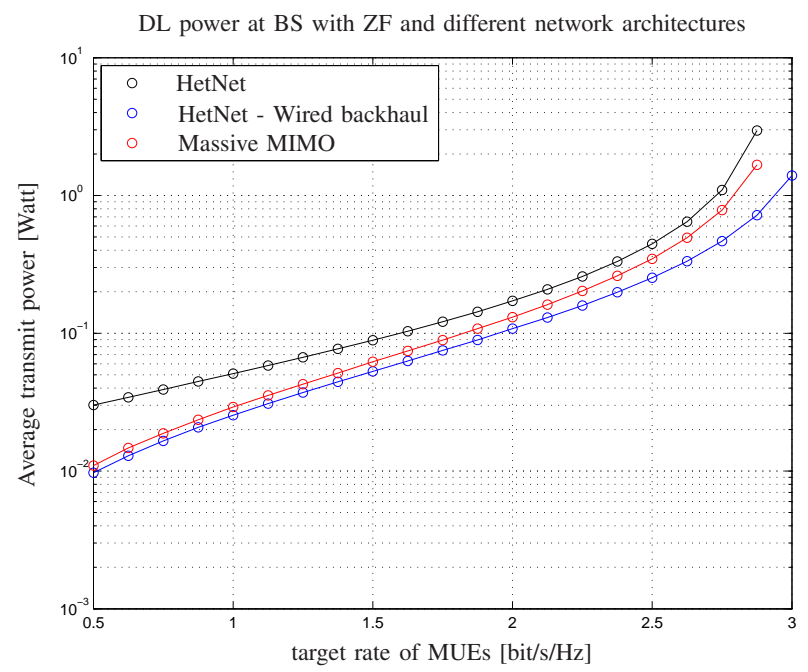

Fig. 6. Average DL transmit power at the BS when ZF is employed with $\tau^{2}=0.1$ and wireless backhaul $3 \mathrm{bit} / \mathrm{s} / \mathrm{Hz}$.

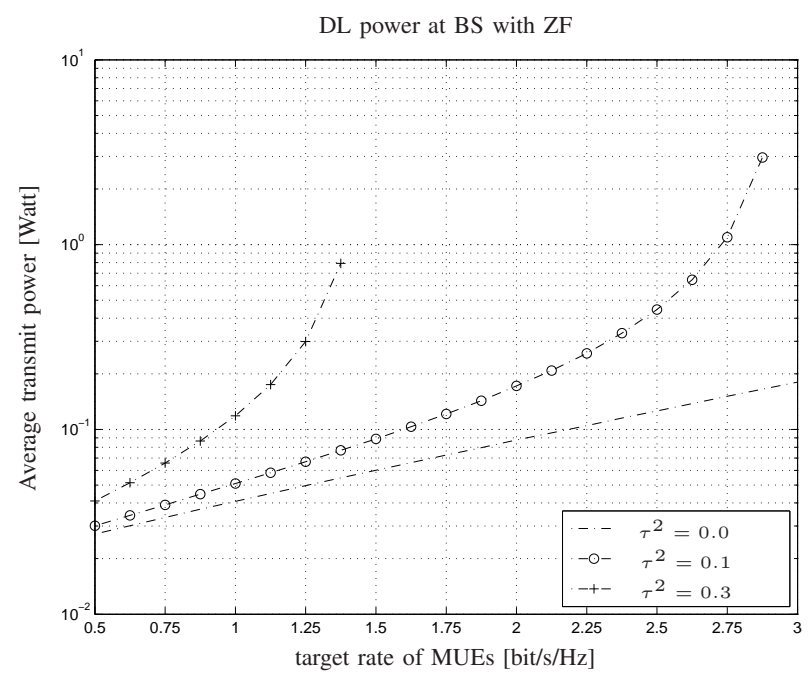

Fig. 7. Average DL transmit power at the BS when $\mathrm{ZF}$ is employed with different values of $\tau^{2}$ and wireless backhaul $3 \mathrm{bit} / \mathrm{s} / \mathrm{Hz}$.

while it is $0.2 \mathrm{~W}$ for a massive MIMO system.

Fig. 7 reports the average DL transmit power at the BS of $\mathrm{ZF}$ when $\tau^{2}=0,0.1$ and 0.3. As seen, for $\tau^{2}=0.3$ the power required diverges when the MUE target rate increases. However, a target rate up to $1.75 \mathrm{bit} / \mathrm{s} / \mathrm{Hz}$ can be handled even with high imperfect CSI.

\section{CONCLUSIONS}

This work has focused on the power consumption in the UL and DL of a HetNet in which a massive MIMO macro tier (serving medium-to-high mobility UEs) is overlaid with a dense tier of SCAs using a wireless backhaul for traffic. A reverse (inter-tier and intra-tier) TDD protocol has been proposed to let the BS simultaneously handle the traffic of macro UEs and SCAs. An MMSE receiver and a concatenated linear precoding technique based on $\mathrm{ZF}$ have been used at the BS for data recovery and transmission while satisfying rate requirements. Results from random matrix theory have been used to derive closed-form expressions for the transmit powers under the assumption of imperfect CSI for mediumto-high mobility UEs. It turns out that for a given set of target rates there is a critical value of imperfect CSI beyond which the power of all transmitters rapidly increases (and eventually diverges). However, analytical and numerical results have shown that when such critical values are not met the proposed architecture allows to achieve an area throughput on the order of a few $\mathrm{Gb} / \mathrm{s} / \mathrm{km}^{2}$ in UL and DL on a $10 \mathrm{MHz}$ band with a very limited amount of power.

\section{REFERENCES}

[1] J. G. Andrews, S. Buzzi, W. Choi, S. Hanly, A. E. Lozano, A. C. K. Soong, and J. C. Zhang, "What will 5G be?" CoRR, vol. abs/1405.2957, 2014. [Online]. Available: http://arxiv.org/abs/1405.2957

[2] B. $\mathrm{Li}$ and P. Liang, "Small cell in-band wireless backhaul in massive MIMO systems: A cooperation of next-generation techniques," CoRR, vol. abs/1402.2603, 2014. [Online]. Available: http://arxiv.org/abs/1402.2603

[3] H. S. Dhillon and G. Caire, "Wireless backhaul networks: Capacity bound, scalability analysis and design guidelines," CoRR, vol. abs/1406.2738, 2014. [Online]. Available: http://arxiv.org/abs/1406.2738

[4] J. Hoydis, K. Hosseini, S. t. Brink, and M. Debbah, "Making smart use of excess antennas: Massive MIMO, small cells, and TDD," Bell Labs Technical Journal, vol. 18, no. 2, pp. 5 - 21, 2013. [Online]. Available: http://dx.doi.org/10.1002/bltj.21602

[5] B. Li and P. Liang, "Small cell in-band wireless backhaul in massive MIMO systems: A cooperation of next-generation techniques," CoRR, vol. abs/1402.2603, 2014

[6] S. Hur, T. Kim, D. Love, J. Krogmeier, T. Thomas, and A. Ghosh, "Millimeter wave beamforming for wireless backhaul and access in small cell networks," IEEE Transactions on Communications, vol. 61, no. 10 , pp. 4391 - 4403, Oct. 2013.

[7] A. Muller, E. Bjornson, R. Couillet, and M. Debbah, "Analysis and management of heterogeneous user mobility in large-scale downlink systems," in Asilomar Conference on Signals, Systems and Computers, Nov. 2013, pp. $773-777$.

[8] H. Shirani-Mehr, G. Caire, and M. Neely, "MIMO downlink scheduling with non-perfect channel state knowledge," IEEE Transactions on Communications, vol. 58, no. 7, pp. 2055 - 2066, July 2010.

[9] P. de Kerret and D. Gesbert, "Degrees of freedom of the network MIMO channel with distributed CSI," IEEE Transactions on Information Theory, vol. 58, no. 11, pp. 6806 - 6824, Nov. 2012.

[10] S. Wagner, R. Couillet, M. Debbah, and D. T. M. Slock, "Large system analysis of linear precoding in correlated MISO broadcast channels under limited feedback," IEEE Transactions on Information Theory, vol. 58, no. 7, pp. 4509-4537, July 2012.

[11] Z. Shen, A. Khoryaev, E. Eriksson, and X. Pan, "Dynamic uplinkdownlink configuration and interference management in TD-LTE," IEEE Commun. Mag., vol. 50, no. 11, pp. 51 - 59, Nov. 2012.

[12] L. Sanguinetti, A. L. Moustakas, and M. Debbah, "Interference management in 5G reverse TDD HetNets: A large system analysis," submitted to J. Sel. Areas Commun., July 2014. [Online]. Available: http://arxiv.org/abs/1407.6481

[13] A. Adhikary, J. Nam, J.-Y. Ahn, and G. Caire, "Joint spatial division and multiplexing - The large-scale array regime," IEEE Transactions on Information Theory, vol. 59, no. 10, pp. 6441 - 6463, Oct 2013.

[14] G. Calcev, D. Chizhik, B. Goransson, S. Howard, H. Huang, A. Kogiantis, A. Molisch, A. Moustakas, D. Reed, and H. Xu, "A wideband spatial channel model for system-wide simulations," IEEE Trans. Veh. Technol., vol. 56, no. 2, pp. 389 - 403, March 2007. 